\title{
Pulmonary epithelial permeability is immediately increased after embolisation with oleic acid but not with neutral fat
}

\author{
JG JONES, BD MINTY, JM BEELEY, D ROYSTON, J CROW, RF GROSSMAN
}

From the Divisions of Anaesthesia and Histopathology, Clinical Research Centre, Northwick Park Hospital, Harrow, and Medical Service, San Francisco General Hospital Medical Center and Cardiovascular Research Institute, University of California, San Francisco, California, USA

ABSTRACT Pulmonary fat embolism occurs frequently after trauma but its functional significance is often unclear. To obtain direct evidence of lung damage caused by fat embolism we have measured changes in permeability of the alveolar-capillary interface. A permeability index was derived from

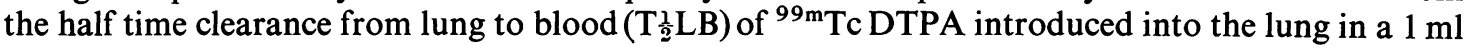
bolus. Three groups of rabbits were studied. Baseline T $\frac{1}{2} \mathrm{LB}$. did not differ significantly between groups. After intravenous injection of saline placebo in one group and of $300 \mathrm{mg} / \mathrm{kg}$ triolein in another group there was no change in permeability index. After intravenous injection of $100 \mathrm{mg} / \mathrm{kg}$ oleic acid in the third group there was an immediate change in $\mathrm{T} \frac{1}{2} \mathrm{LB}$ from a monoexponential baseline $280 \pm 20 \mathrm{~min}(\mathrm{SEM})$ to a multiexponential curve which was resolved into two components, one with a $\mathrm{T} \frac{1}{2} \mathrm{LB}$ of $3.2 \pm 0.6 \mathrm{~min}(\mathrm{SEM})$ and the other $39.5 \pm 7.6 \mathrm{~min}$ (SEM). Statistically significant changes in alveolar-arterial $\mathrm{Po}_{2}$ difference, dynamic compliance, chest radiography, and postmortem lung water accompanied the changes in $T \frac{1}{2} \mathrm{LB}$ in this group. There were no significant changes in these variables in the placebo or triolein group. Histological studies of the lung tissue of these animals using the osmic acid stain for fat showed no fat in the placebo group, extensive fat embolisation which was densely stained in the triolein group and much less densely stained fat in the oleic acid group. Measurement of the permeability of the alveolar-capillary interface provides direct evidence of lung damage after oleic acid embolisation. There were no functional changes in animals with extensive embolisation with triolein.

Fat emboli are frequently found in the lungs of trauma victims ${ }^{12}$ although few of these patients have respiratory symptoms. ${ }^{1}$ However from $30-50 \%$ of patients after trauma have an arterial oxygen tension reduced below $80 \mathrm{mmHg}(11 \mathrm{kPa})$ while breathing air and this has been assumed, without direct evidence, to be caused by subclinical pulmonary fat embolism. ${ }^{1}$ While it is possible that fat embolism may be the cause of a low $\mathrm{PaO}_{2}$ in some patients, the demonstration of hypoxia by itself gives only indirect evidence of lung injury since a fall in $\mathrm{PaO}_{2}$ may also be caused by hypoventilation, breathing at low lung volume, and in some subjects by assuming the supine or Trendelenberg position. Even if fat can be demonstrated clinically in the lung ${ }^{3}$ this need not necessarily be causally related to

Address for reprint requests: Dr JG Jones, Clinical Research Centre, Division of Anaesthesia, Watford Road, Harrow, Middlesex HA1 3UJ. functional impairment because of the different effects of different types of fat on the lung. ${ }^{4}$ In the absence of a specific clinical test for fat in the lung or of its sequelae, in terms of lung dysfunction, we have examined the following hypothesis. Different types of fat embolus may induce measurable differences in the degree of damage to the alveolarcapillary interface and this damage may be measured using a simple radioactive tracer clearance technique.

In this study we have embolised the lung with either neutral fat or oleic acid. The neutral fat used was triolein and was chosen to resemble closely the composition of marrow fat. Oleic acid has been widely used as a model of fat embolism but its effects on pulmonary epithelial permeability have not been studied using the minimally invasive permeability method that we have described.

We have developed a method for measuring pulmonary epithelial damage using the clearance 
rate of $99 \mathrm{~m}$ Tc DTPA (diethylene triamine pentacetate) from lung to blood. ${ }^{5}$ After embolisation of the lungs of animals with either neutral fat or oleic acid we have compared the change in pulmonary epithelial permeability with other changes in lung structure and function. We have shown that the permeability index is a sensitive test of lung damage induced by oleic acid, whereas massive embolisation with neutral fat produced neither change in epithelial permeability nor change in lung function. Thus even if it were possible to demonstrate fat in the lung in vivo, a more important test from the point of view of lung function is the demonstration of damage at the alveolar-capillary interface.

\section{Methods}

New Zealand white rabbits were anaesthetised with an intramuscular injection of $1 \mathrm{ml}$ of Hypnorm ${ }^{\mathrm{R}}$ $(0.2 \mathrm{mg}$ fentanyl base per $\mathrm{ml}$ and $10 \mathrm{mg}$ of fluanisone per $\mathrm{ml}$, Janssen Pharmaceutical), followed $30 \mathrm{~min}$ later by $0.1 \mathrm{ml}$ Hypnorm intravenously into an ear vein. The animals were ventilated with $30 \% \mathrm{O}_{2}$, $70 \% \mathrm{~N}_{2} \mathrm{O}$ in the prone position, via a tracheostomy, using a Harvard small animal ventilator. A catheter was inserted into an ear artery. Tracheal air flow was measured with a heated Fleisch $\mathrm{O}$ pneumotachograph; the flow signal was integrated and displayed on a pen recorder, together with airway pressure (Paw) and arterial pressure. The inspired oxygen concentration was measured with a Servomex paramagnetic oxygen analyser and arterial blood gas tensions with an IL automatic blood gas analyser.

The following measurements were made before and after intravenous injections of either neutral fat, oleic acid, or saline placebo: pulmonary epithelial permeability; alveolar-arterial oxygen gradient; dynamic compliance; lung radiographic score.

Postmortem measurements were made of lung wet weight/bloodless dry weight ratio and in a separate group of animals the lungs were fixed in formalin and used to stain for the presence of fat.

\section{PULMONARY EPITHELIAL PERMEABILITY}

An index of pulmonary epithelial permeability was derived from the half time clearance rate of 99mTc DTPA (MW 492 Daltons) from lung into blood. This technique is similar to that previously described for use in man, ${ }^{56}$ although in animals the tracer is introduced into the lung in a small bolus. ${ }^{7}$ Two collimated miniature scintillation detectors were positioned, one over the lungs and one over the head. A $1 \mathrm{ml}$ bolus of $99 \mathrm{~m}$ Tc DTPA in saline was injected into the lung via a fine catheter whose tip was located at the carina. This was followed by $0.5 \mathrm{ml}$ of saline and $1 \mathrm{ml}$ of air. The lung detector was moved until a maximum reading was obtained and counts from the lung and head were obtained for 20 second epochs every minute for the duration of the study. The lung detector recorded activity from part of lung and vascular tissues in its field of view. The increase in tissue background radioactivity in the field of this detector was corrected, using the signal from the head detector in the following manner. Towards the end of the study period the animal was given an intravenous injection of $20 \mu \mathrm{Ci}$ of $99 \mathrm{mTc}$ DTPA. This produced an increase in signal in both head and lung detector proportional to the radioactivity in the vascular tissues in the two detector fields. The ratio of the increase in count rate in the lung field to the increase in count rate in the head field was calculated. For each minute after tracheal instillation of the tracer, the head count rate was multiplied by this ratio and the product subtracted from the lung count rate. The resulting lung to blood clearance curve represented the rate of declining activity in the lung alone and the time for this corrected curve to fall to half the peak activity was used as an index of pulmonary epithelial permeability ( $\left.\mathrm{T} \frac{1}{2} \mathrm{LB}\right)$.

\section{ALVEOLAR-ARTERIAL OXYGEN TENSION DIFFERENCE (A-aPO2)}

The alveolar-arterial oxygen tension difference was calculated from the arterial blood gas tensions and the inspired oxygen concentration assuming a respiratory quotient of $0 \cdot 8$.

DYNAMIC COMPLIANCE

Dynamic compliance (Cdyn) was derived from the tidal volume and change in Paw between points of zero flow.

\section{LUNG RADIOGRAPHS}

Lung radiographs were obtained with the lungs transiently inflated to a transpulmonary pressure of $20 \mathrm{~cm} \mathrm{H}_{2} \mathrm{O}$ at the time of exposure. The technique used was $60 \mathrm{kV}, 130 \mathrm{~mA}$ with 0.045 exposure using a $1 \mathrm{~mm}$ focal spot. Radiographs were scored for pulmonary oedema according to the following scale: $\mathbf{0}=$ distinct vascular markings with no infiltrates; $1=$ scattered punctate infiltrates no greater than $0.2 \mathrm{~cm}$ in diameter; $2=$ discrete infiltrates less than $1 \mathrm{~cm}^{2}$ in area with blurring of vascular markings; $3=$ confluent infiltrates greater than $1 \mathrm{~cm}^{2}$ in area.

POSTMORTEM LUNG STUDIES

Lung wet weight to bloodless dry weight ratio was calculated using a modification of the method of Pearce, Yamashita and Beazell. ${ }^{8} \mathrm{~A}$ separate group of animals was used for histological study. The tissues were block-stained with osmium tetroxide, 
embedded in wax and sections counterstained with neutral red.

\section{PROCEDURE}

With the animals anaesthetised and mechanically ventilated baseline measurements were made of $\mathrm{A}-\mathrm{aPo}_{2}$ difference, Cdyn, and chest radiographic score. Then ${ }^{99 \mathrm{~m} T c}$ DTPA was instilled into the airway and the clearance curve was constructed. Measurements of $\mathrm{A}-\mathrm{aPO}_{2}$ difference, Cdyn, and chest radiographic score were repeated 30 minutes after isotope instillation. At this time the $\mathrm{A}-\mathrm{aPO}_{2}$ and Cdyn had returned to baseline and the animals were divided into three groups: group $1(\mathrm{n}=8)$ was given oleic acid intravenously (100 $\mathrm{mg} / \mathrm{kg}$ body wt); group 2 $(\mathrm{n}=8)$ was given triolein intravenously $(99 \%$ in sealed ampoules refrigerated under nitrogen, Sigma Ltd) $(300 \mathrm{mg} / \mathrm{kg}$ body wt $)$; and group $3(\mathrm{n}=6)$ was given a comparable volume of saline intravenously. Repeated measurements of $\mathrm{A}-\mathrm{aPo}_{2}$ difference, Cdyn, and chest radiograph were then made 15,30 , and 45 minutes after "fat" or placebo injection. The ${ }^{99 \mathrm{~m} T c}$ DTPA clearance was measured throughout the duration of the study.

After calibration for contribution of tissue radioactivity to the lung detector the animals were killed with intravenous barbiturate and lungs either fixed in formalin and stained for fat or studied for extravascular lung water content.

\section{Results}

\section{PERMEABILITY INDEX}

During the baseline period before the injection of "fat" or placebo there was no significant difference in the clearance rate, expressed as $\mathrm{T}_{\frac{1}{2}} \mathrm{LB}$, in the three groups of animals (table). This clearance curve was almost invariably monoexponential except after embolisation with oleic acid when it could always be resolved into two clearly defined exponential curves. After the injection of oleic acid in the group 1 animals there was always a rapid increase in the clearance of $99 \mathrm{~m}$ Tc DTPA. In neither group 2 (triolein) or group 3 (placebo) was there any significant change in slope of the clearance curve. Three typical studies are shown in fig 1. The dramatic change in slope of the curve in animals given oleic acid could be resolved into an initial very rapid component, mean $T_{\frac{1}{2}} 3.2 \pm 0.6 \mathrm{~min}$ (SEM), and a slower second component, mean $T_{\frac{1}{2}} 39.5 \pm 7.6$ min (SEM). Extrapolation of these curves to the time of injection showed that the fast component corresponded to a compartment containing $24.4 \pm$ $3.8 \%$ of the radioactivity in the lung.

A-aPO2 DIFFERENCE

Data for the three groups of animals are shown
Table Mean clearance rate of ${ }^{99 m}$ Tc DTPA from the lung, expressed as $T_{\frac{1}{2}} L B$ in minutes ( $\left.\pm S E M\right)$. The animals were divided into three groups, the baseline $T_{\frac{1}{2}} L B$ in each group was not significantly different. The effect on $T \frac{1}{2} L B$ of intravenous injection of placebo, triolein, or oleic acid in each group is shown. The $T \frac{1}{2} L B$ values after oleic acid represent the two exponentials always seen in this group

\begin{tabular}{|c|c|c|c|}
\hline & & \multicolumn{2}{|c|}{$\begin{array}{l}\text { Half time clearance from lung to blood } \mathrm{min} \\
(S E M)\end{array}$} \\
\hline Group 3 & $\begin{array}{l}n \\
6\end{array}$ & $\begin{array}{l}\text { Baseline } \\
260 \pm 50\end{array}$ & $\begin{array}{l}\text { Post placebo } \\
252 \pm 55 \\
\text { Triolein }\end{array}$ \\
\hline Group 2 & 8 & $246 \pm 63$ & $\begin{array}{l}237 \pm 50 \\
\text { Oleic acid }\end{array}$ \\
\hline Group 1 & 8 & $280 \pm 20$ & $3.2 \pm 0.6 ; 39.5 \pm 7.6$ \\
\hline
\end{tabular}
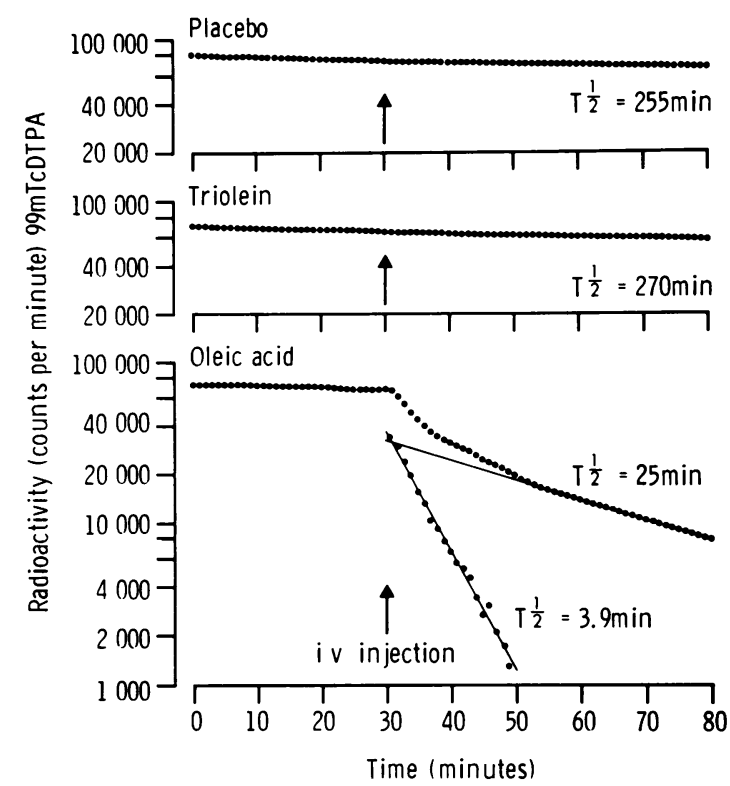

Fig 1 Lung clearance curve from one animal in each group given either placebo, triolein, or oleic acid intravenously. The time of injection is shown by the arrow. After injection of oleic acid the curve was resolved into two components.

in fig 2. There was no significant change in $\mathrm{A}-\mathrm{aPo}_{2}$ difference after the placebo and triolein injection. After the oleic acid injection there was a statistically significant $(\mathrm{p}<0.001)$ increase in $\mathrm{A}-\mathrm{aPo}_{2}$ difference from 15 minutes after injection.

DYNAMIC COMPLIANCE

Data for the three groups of animals are shown in fig 3. There was no significant change in Cdyn for the placebo and triolein groups. The oleic acid 


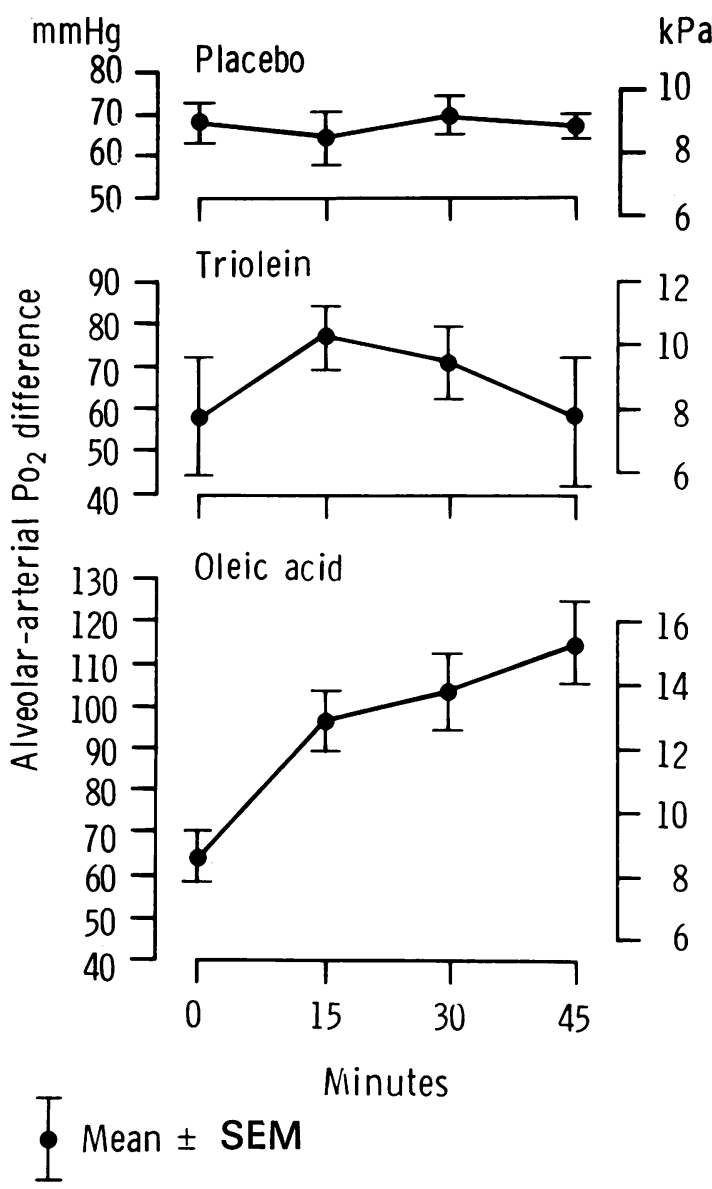

Fig 2 Values for $\mathrm{A}-\mathrm{aPo}_{2}$ difference in the three groups of animals after intravenous injection of either placebo, triolein, or oleic acid.

group showed a statistically significant $(\mathrm{p}<0.001)$ fall in Cdyn from 15 minutes after injection.

\section{LUNG RADIOGRAPHS}

There were no changes in the pulmonary oedema score of the animals given either placebo or triolein. The changes in score of the oleic group are shown in fig 4. Changes compatible with pulmonary oedema were evident at 15 minutes after injection. The scores, analysed by the Rank-Wilcoxon method, were significantly different $(p<0.001)$ from baseline scores.

\section{POSTMORTEM ANALYSIS OF WET/DRY LUNG} WEIGHT

The placebo group had a bloodless lung wet weight to dry weight ratio of $4 \cdot 0 \pm 0 \cdot 1$ (SEM), the triolein

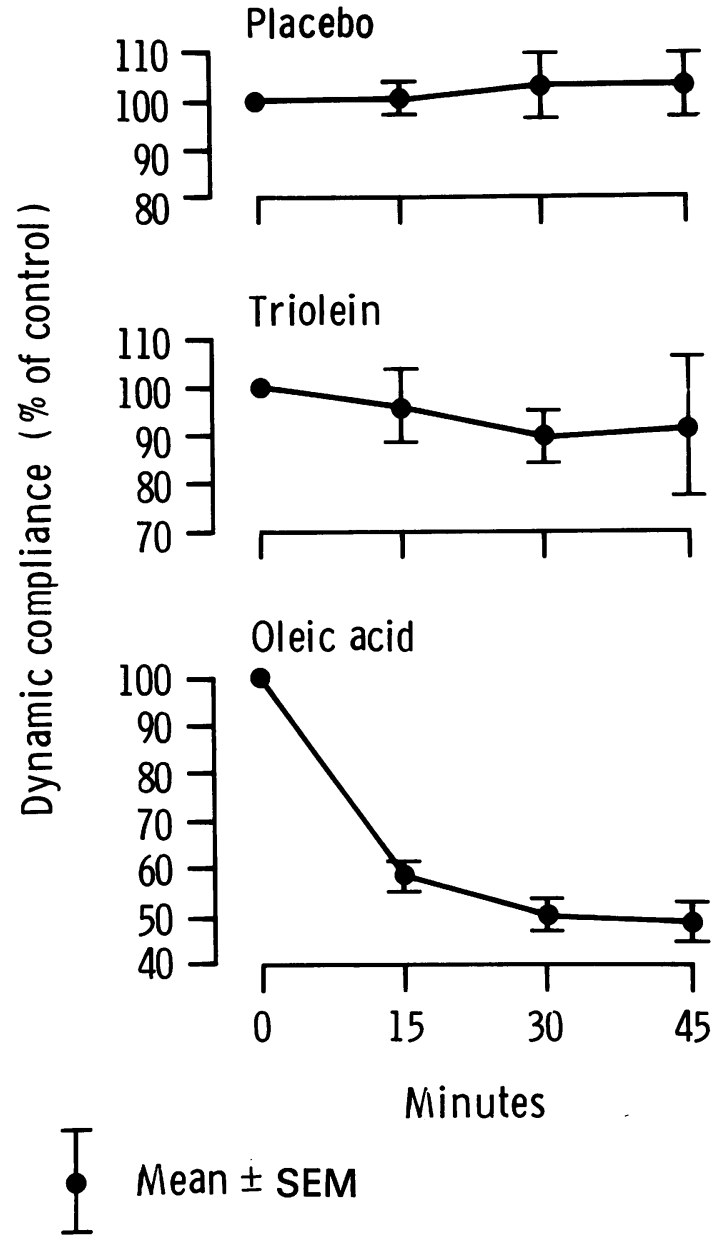

Fig 3 Dynamic compliance (expressed as \% of control) in the three groups of animals.

group $4.41 \pm 0.16$ and the oleic acid group $5 \cdot 1 \pm$ $0 \cdot 1$. The lung water in the triolein group was not significantly greater than that in the placebo group whereas the lung water in the oleic acid group was significantly $(p<0.001)$ greater than in the placebo group.

\section{HISTOLOGY}

The histological sections were presented to the pathologist without indicating from which group of animals they came. The most widespread and dense staining was found in the triolein group (fig 5a). The oleic acid group showed much less staining for fat and the appearance of the staining was "smudgy" and less well defined than that seen in the triolein animals (fig $5 b$ ). The placebo group showed no evidence of fat staining. 


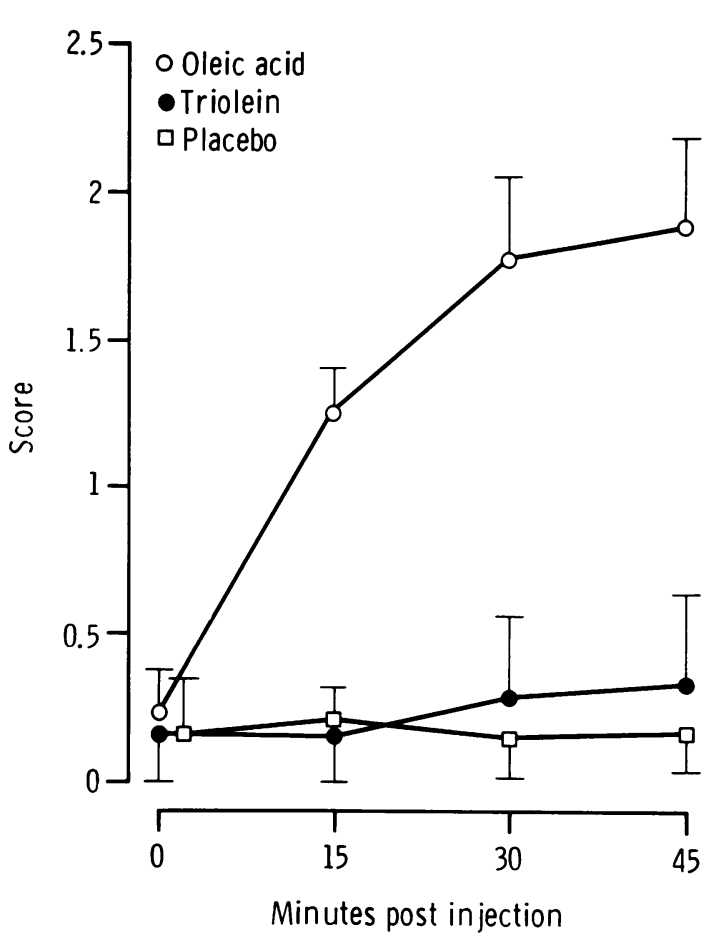

Fig 4 Mean $\pm S E M$ radiographic score for pulmonary oedema in the three groups of animals.

\section{Discussion}

Using a modification of a simple test ${ }^{5}$ of the permeability of the alveolar epithelial membrane, we have shown quite characteristic types of effects on the lungs in our two groups of "fat" embolism animals. After intravenous injection of oleic acid the abrupt increase in permeability was in complete contrast to the absence of effect after intravenous triolein or placebo. The pattern of lung damage after oleic acid, measured from the $99 \mathrm{~m}$ Tc DTPA clearance curve, suggested that about $24 \%$ of the lung containing radioactive tracer was very severely damaged, the T1 1 LB falling from a control of $280 \mathrm{~min}$ to $3 \cdot 2$ min while the remainder of the lung was much less severely damaged. It is probable that the alveoli with the very short $T_{\frac{1}{2}}$ LB were the major site of leakage of plasma through the alveolar capillary interface. This alveolar flooding produced a significant functional impairment, measured from gas exchange and compliance data, extensive radiological changes and an increase in postmortem bloodless lung water. The large difference in the permeability index between the oleic acid group on the one hand and the triolein and placebo groups

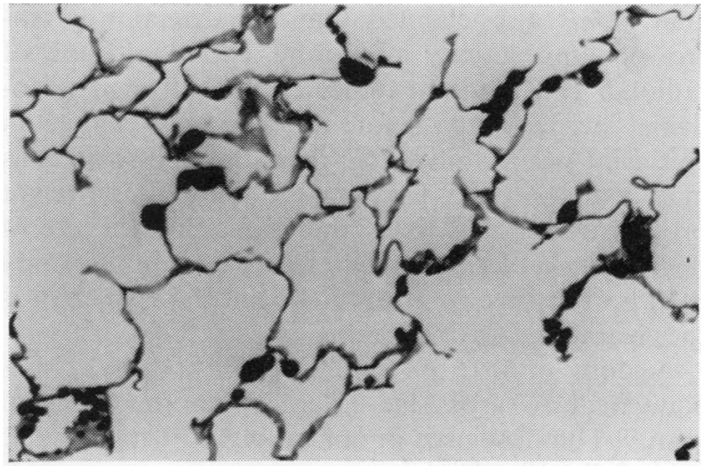

(a)

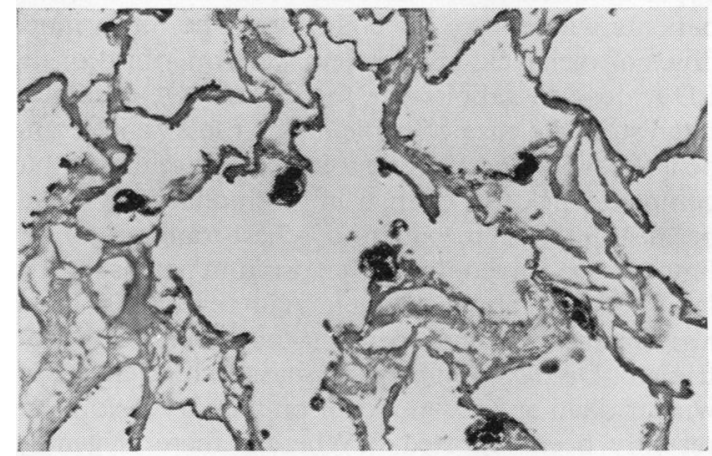

(b)

Fig 5 (a) Histological appearance of fat in an animal given triolein, stained with osmium tetroxide and neutral red $(\times 283)$; (b) smudgy appearance of fat seen after oleic acid, stained with osmium tetroxide and neutral red $(\times 283)$.

on the other, was consistent with the large functional, radiological, and lung water content differences between these groups. This suggests that the permeability index, derived using a small hydrophilic tracer molecule, is a very useful screening test of the integrity of the alveolar-capillary interface and if the $\mathrm{T} \frac{1}{2} \mathrm{LB}$ is very short ( $\left.3 \mathrm{~min}\right)$, it may predict the likelihood of pulmonary oedema. Derivation of this index could be of value in the clinical situation to differentiate functional derangements caused by fat embolism from those from other causes or from preexisting chronic lung disease. A similar effect on lung permeability has been demonstrated by Sugerman et $a l^{9}$ with oleic acid using intravenously injected radiolabelled albumin detected in the lung interstitium with the Gamma camera. They also suggested that their technique was a more sensitive indicator of lung damage than changes in $\mathrm{PaO}_{2}$ or chest radiographs. In this regard it was notable that one of our oleic acid animals showed a decrease in T $\frac{1}{2}$ LB without impairment of gas exchange. Suger- 
man et $a l^{9}$ also claimed that the detection of increased lung activity after intravenous injection of radiolabelled albumin indicated a change in endothelial permeability before there was a change in epithelial permeability to this molecule. While this may well be true for a large molecular weight protein tracer, it is likely that the clearance rate of the smaller molecular weight tracer used in our study is more sensitive to early epithelial damage than is the clearance rate of albumin.

A dose of $0.15 \mathrm{ml}$ of fat $/ \mathrm{kg}$ of body weight is equivalent to gross fat embolism in the lungs of man. ${ }^{10}$ The dose used in our rabbits $(0.1 \mathrm{ml} / \mathrm{kg}$ oleic acid, $0.3 \mathrm{ml} / \mathrm{kg}$ triolein) may thus be a reasonable approximation to the amount of fat in the lungs of patients with severe fat embolism. The intravenous doses of oleic acid and triolein were one-third of the LD $_{50}$ dose in rabbits for these fats. ${ }^{4}$ Of particular interest in the case of triolein was the extensive and densely staining fat on histological examination but complete lack of change in pulmonary epithelial permeability or in function, chest radiograph, or lung water. In contrast the osmium stains of the lungs of animals given oleic acid revealed a much less distinct staining pattern than that seen with triolein. Dissociation between massive lung embolism with triolein and insignificant pulmonary lesions has recently been reported. ${ }^{11}$ Whereas there is general agreement about the effects on the lung of oleic acid, 491213 there is some conflict in the literature regarding the effects of intravenous triolein or neutral fat. Some authors have shown no significant change on light or electron microscopy in the lungs of animals given triolein. ${ }^{14}$ Others have shown that neutral fat or triolein induces interstitial oedema and changes in light and electron microscopic appearance. ${ }^{15} 16$ Some of these differences may be attributed to free fatty acid contamination of the neutral fat. Triolein kept refrigerated under nitrogen, as was the case in this study, is free of fatty acid contamination and is acceptable in studies of the effects of pure neutral fat in the lung. The effect of embolisation with neutral fat may induce lung damage at a very much slower rate than that seen with oleic acid. ${ }^{4}$ This may be the result of the slow rate of hydrolysis of neutral fat to short chain fatty acids. The time course of our study was too short to demonstrate this effect because we were interested only in the immediate responses to fat embolism.

The clinical implications of these observations are as follows. Extensive pulmonary embolisation with marrow fat may be quite innocuous if the rate of hydrolysis to short chain fatty acid is slow. Sequential measurement of $\mathrm{T} \frac{1}{2} \mathrm{LB}$ using the technique that we have developed for use in $\operatorname{man}^{5}$ may show a progressive reduction in $T \frac{1}{2}$ LB if toxic fatty acids are being released by hydrolysis of neutral fat emboli in significant amounts in the lung.

We conclude that ${ }^{99 \mathrm{~m}} \mathrm{Tc}$ DTPA clearance from the lung is a very sensitive indicator of pulmonary epithelial damage in fat embolism. The dense staining of neutral fat, using the osmium tetroxide technique, and the absence of functional or structural effect is compatible with the view that histological fat is common but that severe functional effects are rare.

This work was supported in part by NHLBI grant HL-19155 (Pulmonary vascular disease SCOR).

\section{References}

${ }^{1}$ Sevitt S. The significance of fat embolism. Br J Hosp Med 1973;9:784-93.

2 Pietra GG, Rüttner JR, Wüst W, Glinz W. The lung after trauma and shock-fine structure of the alveolarcapillary barrier in 23 autopsies. J Trauma 1981;21: 454-62.

${ }^{3}$ Ellison LT, McPherson JC, Anabtawi IN, Ellison RG. Incidence of free fat in the lung during open-heart surgery. Ann Thorac Surg 1969;7:509-17.

${ }^{4}$ Peltier LF. Fat Embolism III. The toxic properties of neutral fat and free fatty acids. Surgery 1956;40:665-70.

5 Jones JG, Minty BD, Lawler P, Hulands G, Crawley JCW, Veall N. Increased alveolar epithelial permeability in cigarette smokers. Lancet $1980 ; 1$ :66-8.

${ }^{6}$ Minty B, Jordan C, Jones JG. Rapid improvement in abnormal pulmonary epithelial permeability after stopping cigarettes. Br Med J 1981 ;282:1183-6.

' Jones JG, Berry M, Hulands GH, Crawley JCW. The time course and degree of change in alveolar-capillary membrane permeability induced by aspiration of hydrochloric acid and hypotonic saline. Am Rev Respir Dis 1978;118:1007-13.

${ }^{8}$ Pearce ML, Yamashita J, Beazell J. Measurement of pulmonary edema. Circ Res $1965 ; 16: 482-8$.

${ }^{9}$ Sugerman HJ, Strash AM, Hirsch JI, et al. Sensitivity of scintigraphy for detection of pulmonary capillary albumin leak in canine oleic acid ARDS. J Trauma $1981 ; 21$ :520-7.

${ }^{10}$ Armin J, Grant RT. Observations on gross pulmonary embolism in man and rabbit. Clin Sci 1951;10:441-69.

11 Jacobovitz-Derks D, Derks CM. Pulmonary neutral fat embolism in dogs. Am J Pathol 1979;95:29-38.

12 Derks CM, Jacobovitz-Derks D. Embolic pneumopathy induced by oleic acid. A systematic morphologic study. Am J Pathol 1977;87:143-58.

${ }^{13}$ Delaunois L, Sergysels R, Martin RR. Acute effects on airways mechanics of pulmonary edema induced by intravenous oleic acid in dogs. Bull Eur Physiopathol Respir 1980;16:47-55.

14 Jacobs RR, Wheeler EJ, Jelenko C, McDonald TF, Bliven FE. Fat embolism: a microscopic and ultrastructure evaluation of two animal models. J Trauma 1973;13:980-93.

${ }^{15}$ Reidbord HE. Pulmonary fat embolism. An ultrastructure study. Arch Pathol 1974;98:122-5.

${ }^{16}$ Shaffer JW, Sealy WC, Seaber AV, Goldner JL. Etiology of fat embolism syndrome: Early morphologic lung changes of respiratory distress syndrome produced by triolein. Surg Forum 1976;27:516-8. 